\title{
Contralateral recurrence of aggressive fibromatosis in a young woman: A case report and review of the literature
}

\author{
CHRISTOPHER J. SCHMOYER, HARMAR D. BRERETON and ERIC W. BLOMAIN
}

Clinical Faculty, Department of Medicine, The Commonwealth Medical College, Scranton, PA 18509, USA

Received August 9, 2014; Accepted April 24, 2015

DOI: $10.3892 / 01.2015 .3215$

\begin{abstract}
Aggressive fibromatosis (AF) is a benign non-encapsulated tumor of mesenchymal origin, with a tendency for local spread along fascial planes. Local invasion can lead to extensive morbidity and even mortality due to destruction of the bones, organs and soft tissues. This rare lesion is observed 1,000 times more frequently in patients with familial adenomatous polyposis or Gardner's syndrome due to the inheritance of the adenomatous polyposis coli $(A P C)$ gene. While AF does not metastasize, local recurrence is common. Distant recurrence is extremely rare, but is observed in those with a germ line $A P C$ mutation. The present study details the case of a 20-year-old woman with a melanoma of the right shoulder, treated definitively with surgery. The patient then developed a painful mass at the surgical site; a surgical biopsy demonstrated that the mass was AF. The patient was treated with surgical resection, radiation therapy, and a course of tamoxifen. Five years later, the patient presented with left forearm pain and diminished range of motion due to an infiltrating mass. This was excised and a clinical diagnosis of recurrent $\mathrm{AF}$ was made, in this patient lacking familial predisposition to the disease.
\end{abstract}

\section{Introduction}

Aggressive fibromatosis (AF), first identified by Stout in 1954 (1), is a benign non-encapsulated lesion of mesenchymal origin, with a tendency for local spread along fascial planes. AF is locally invasive to bones, organs and other tissues, which can be fatal. The tumors are rare, observed in 2-4 cases per million individuals each year (2), and typically occur between puberty and the age of 40 , with a slight female preponderance. AF can arise in the musculoaponeurotic tissue of any location, but is common in the abdominal wall, extremities, head and neck,

Correspondence to: Mr. Christopher J. Schmoyer, Clinical Faculty, Department of Medicine, The Commonwealth Medical College, 525 Pine Street, Scranton, PA 18509, USA

E-mail: schmoyerc@gmail.com

Key words: aggressive fibromatosis, recurrence, case report, contralateral, oncology and shoulder girdle. Individuals with familial adenomatous polyposis (FAP) or Gardner's syndrome have a 1,000 times greater risk for developing the disease due to inheritance of the adenomatous polyposis coli (APC) gene (3). These patients may present with intra-abdominal lesions following colonic resection (4). While AF does not metastasize, local recurrence is common. Distant recurrence is extremely rare, but is typically observed in those with a new primary tumor associated with the $A P C$ mutation. The present study reports the case of a 20 -year-old female with sporadic contralateral recurrence of clinically diagnosed AF and no familial predisposition.

\section{Case report}

A 20-year-old female first presented in April 1992 at Northeastern Pennsylvania Plastic Surgery Associates (Scranton, PA, USA) with a pigmented lesion on the right shoulder overlying the trapezius. The lesion had undergone a recent change in size and color. Upon physical examination, an irregularly contoured brown papule with portions of jet black speckling was noted. No cervical or axillary adenopathy was present. The past medical history was significant for a mitral valve prolapse and asthma. The patient's family history was free of any familial disease. A biopsy of the lesion was performed, which confirmed a 0.4-mm thick malignant melanoma, Clark's level III. The patient underwent a negative staging workup and the $1.7-\mathrm{cm}$ tumor was excised with plastic reconstruction. The margins were negative.

One year later, in July 1993, the patient returned to Northeastern Pennsylvania Plastic Surgery Associates complaining of pain at the surgical site. The area was profoundly painful upon palpation and a discrete mass was identified deep to the incision (Fig. 1A). Two enlarged axillary lymph nodes were noted. Fearing a recurrent melanoma, magnetic resonance imaging (MRI) and biopsies of the lymph nodes and shoulder mass were obtained. MRI showed a $7 \times 3 \times 3-\mathrm{cm}$ area of altered signal extending from the skin into the trapezius muscle. The lesion was isodense to muscle on T1-weighted imaging, with increased intensity on T2-weighted imaging (Fig. 1B). On biopsy, the nodes were benign and reactive in nature. The lesion itself showed a proliferation of spindle cells, with no evidence of increased mitotic rate or necrosis, in a collagenous matrix infiltrating into the adjacent muscle fibers (Fig. 1C). S-100 and HMB-45 stains were negative. A diagnosis of AF was made and confirmed by independent pathologists at the 
A

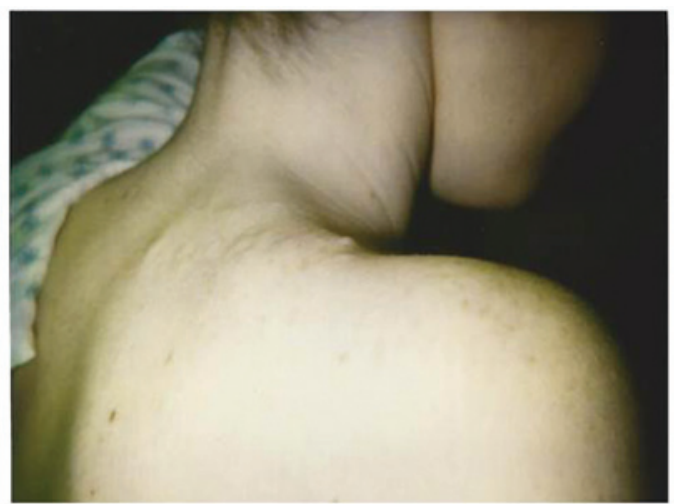

B

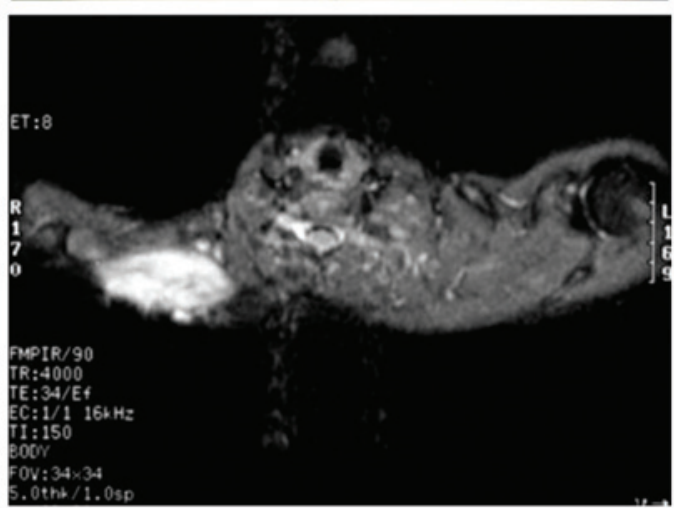

C

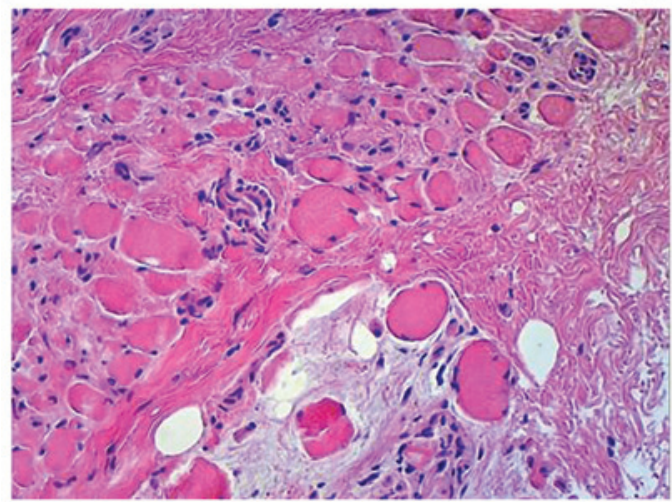

Figure 1. Right shoulder fibromatosis. (A) Palpable mass present at the previous surgical site. (B) Axial fluid-sensitive pulse sequence fat-suppressed T2-weighted magnetic resonance image. (C) Hematoxylin and eosin staining showing fibrotic tissue with bland spindle cells invading atrophic skeletal muscle fibers, with surrounding myxoid change, x200 magnification.

University of Pennsylvania (Philadelphia, PA, USA) and the Roswell Park Cancer Institute (Buffalo, NY, USA) (Fig. 1A). The patient initially refused surgery or radiation therapy and was treated with tamoxifen. Tamoxifen was started at $20 \mathrm{mg}$ once daily beginning in June 1995 following recovery from surgery and radiation treatments No improvement was noted and the patient eventually authorized surgical excision due to the rapid growth and infiltrative properties of the lesion. The mass was resected along with the right trapezius muscle due to tumor invasion and the shoulder girdle was resuspended with the levator scapulae muscle. Due to a positive margin, the patient was referred for radiation therapy and tamoxifen treatment was continued: Tamoxifen treatment continued at $20 \mathrm{mg}$ once daily until January 1996, when the treatment was discontinued at the request of the patient. Radiotherapy consisted of 4,500 cGy in 180-cGy fractions, with $6 \mathrm{MeV}$ photons to the

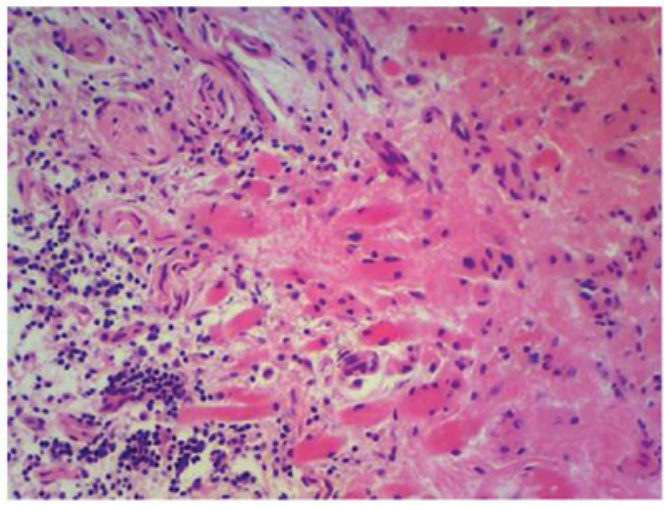

Figure 2. Hematoxylin and eosin staining of the left forearm lesion showing atrophic muscle fibers with interdigitating fibrosis and myxoid change, $\mathrm{x} 200$ magnification.

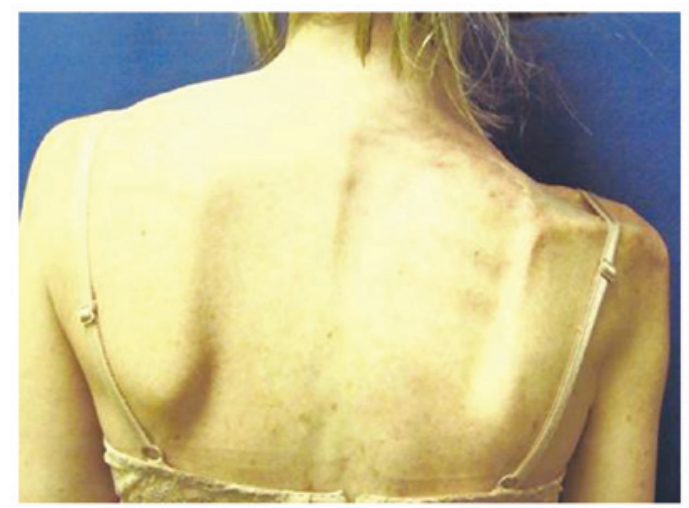

Figure 3. Depression of the scapula due to levator scapulae shoulder girdle resuspension, with post-radiation dermal changes.

right shoulder and scapula. A boost of 1,620 cGy in 180-cGy fractions, with $9 \mathrm{MeV}$ electrons, was administered to the surrounding scar. The treatment was successful and follow up MRI scans showed no evidence of recurrent disease.

Nearly 5 years after the diagnosis of right shoulder AF, in March 1999, the patient presented at Northeastern Pennsylvania Plastic Surgery Associate with left forearm pain and failure to pronate or supinate the extremity $>10^{\circ}$. Flexion and extension were preserved. There was exquisite tenderness upon palpation between the radius and ulna proximally. Upper extremity sensation was intact and reflexes were $2+$ bilaterally using the National Institute of Neurological Disordersand Stroke (NINDS) scale developed by Hallett in 1993 (5). An MRI scan was obtained showing a thin 5-cm area of enhancement located between the proximal radius and ulna, possibly involving the periosteum of each. The area was isodense to muscle on T1-weighted imaging, with increased intensity on T2-weighted imaging. A monophasic bone scan showed areas of increased intensity in the proximal radius and ulna. A computed tomography-guided needle biopsy demonstrated a collagenous fibrous stroma with plump spindle cells devoid of increased mitotic activity, consistent with AF. The patient underwent surgical resection of the forearm lesion, but declined further radiation or medical therapy. Although grossly and clinically the lesion behaved as AF, the final pathological report was equivocal, noting fibroadipose tissue with myxoid 
change (Fig. 2). However, a clinical diagnosis of AF was made and the patient was treated accordingly.

The patient still attends follow-up examinations nearly 20 years after the initial diagnosis of melanoma. The patient is in a good condition and has experienced no further recurrence of either lesion. However, shoulder girdle suspension using the levator scapulae is a continual source of pain and weakness, and may require further stabilization in the future (Fig. 3).

\section{Discussion}

Sporadic AF arises from somatic mutations in the $A P C$ gene or in $C T N N B 1$, which codes for $\beta$-catenin $(6,7)$. Germline $A P C$ mutations found in individuals with FAP predispose the patient to AF. APC acts to downregulate the expression of $\beta$-catenin as part of the canonical Wnt pathway. In the absence of activation, a complex of APC, axin and glycogen synthase kinase $3 \beta$ phosphorylates $\beta$-catenin resulting in ubiquitin-mediated degradation (8). Mutation prevents this complex from forming. $\beta$-catenin accumulates in the cytoplasm and nucleus where it subsequently binds transcription factors of the T cell/lymphoid enhancer-binding factor family, inducing gene expression (9). Hedgehog signaling, which modulates the Wnt pathway and $\beta$-catenin expression, is also involved in the pathogenesis (10). The buildup of $\beta$-catenin within mesenchymal progenitor cells likely maintains an undifferentiated fibroblast-like state, producing uncontrolled proliferation and stromal expansion (11).

The tumor exhibits phases of progression, latency and occasional spontaneous regression. Extra-abdominal tumors produce symptoms, including pain, weakness and parasthesia. Intra-abdominal lesions can cause intestinal obstruction or ischemia (12). MRI is the most useful imaging technique for diagnosis, showing intermediate intensity on T1-weighted imaging and high signal intensity on T2-weighted imaging. Intravenous contrast agents produce moderate- to high-grade enhancement in highly cellular regions. Non-enhancing bands of low intensity dominate, representing densely packed fibrous tissue $(13,14)$. Once AF is identified, a tissue biopsy should be performed. Fine-needle aspiration is acceptable if an open biopsy is contraindicated (15). Histological analysis shows a scattered proliferation of spindle cells with bland, occasionally bipolar, nuclei. Neoplastic cells are dispersed among a dense collagenous, keloid-like or myxoid matrix. Staining for nuclear $\beta$-catenin is positive, while staining for S-100, c-kit, cluster of differentiation 34, estrogen receptor $\alpha$ and desmin is negative (15). A cytogenic association with trisomy 8 and 20 is occasionally noted (16).

For stable or slow growing masses, close monitoring is now advocated as the primary treatment strategy, as AF has a tendency to regress. Studies have shown no difference in survival between patients treated with surgery or radiation therapy compared with no treatment in this population $(17,18)$. Surgical resection is indicated in those individuals with aggressive lesions. The 5-year risk of recurrence following resection is $17.6 \%$, regardless of whether or not microscopic margins remain (19). Radiation therapy at a dose of 50-60 Gy is also an effective primary therapy (20). Neoadjuvant or adjuvant radiation is occasionally performed, but its use is controversial. Conflicting studies have shown either no difference in recurrence rate following adjuvant therapy (21) or a delayed time to recurrence with no impact on overall survival (22). Patients with contraindications to surgery or radiotherapy can undergo medical treatment. Non-steroidal anti-inflammatory drug therapy with sulindac or indomethacin has proven effective due to tumor overexpression of cyclooxygenase-2 (23). A number of small trials and case reports indicate that anti-estrogen therapy, including tamoxifen or toremifine, appears to improve or stabilize AF lesions (24). A large randomized trial is required to corroborate these reports. Systemic chemotherapy also may be used, although specific guidelines are lacking. Tyrosine kinase inhibitors or anthracyclines are popular, but the use of a variety of other agents is possible (25).

The present study reports a case with a presumptive recurrence of $\mathrm{AF}$ at a site distant and contralateral to the initial lesion. Local recurrence of AF is common and is usually caused by residual tumor cells following treatment failure. There have also been reports of patients with local multicentric tumors present at diagnosis. These include a patient with masses on the right thigh and buttock (26), and another with masses in the left hip and popliteal fossa (27). In these cases, microscopic contiguous invasion of the initial lesion probably led to the formation of the second focus. Even rarer are reports of distant recurrence occurring on the same side of the body. A 2002 report by Watanabe et al reported the case of a patient with AF of the right dorsal foot. The tumor recurred on the right knee, followed later by the right thigh and right shoulder (28). To the best of our knowledge, there has been only one published case of AF present on contralateral sides of the body. Contralateral lesions were found in multiple members of a family with FAP secondary to an inherited $A P C$ gene mutation. The index case presented with multifocal AF of the paraspinal muscles, which later recurred intra-abdominally. Affected relatives include a woman with recurrent $\mathrm{AF}$ of the right occiput and bilateral breasts, as well as a male with cutaneous AF of each arm, the right occiput and multiple paraspinal masses (29). In this previous study, the germline $A P C$ gene mutation predisposed the family to form multiple AF lesions. The patient described in the present study, however, had no family history of AF or FAP. It is likely that the clinically diagnosed contralateral distant recurrence was the result of the development of a second primary tumor. Contiguous spread from the initial site on the right shoulder to the left forearm five years later is improbable.

Contralateral recurrence of AF is extremely rare. However, a low index of suspicion for recurrent AF should be maintained in patients presenting with symptoms of muscle weakness, pain, parasthesia and a palpable mass.

\section{References}

1. Stout AP: Juvenile fibromatoses. Cancer 7: 953-978, 1954.

2. Hosalkar HS, Torbert JT, Fox EJ, Delaney TF, Aboulafia AJ and Lackman RD: Musculoskeletal desmoid tumors. J Am Acad Orthop Surg 16: 188-198, 2008.

3. Gurbuz AK, Giardiello FM, Petersen GM, et al: Desmoid tumours in familial adenomatous polyposis. Gut 35 : 377-381, 1994.

4. Hartley JE, Church JM, Gupta S, McGannon E and Fazio VW: Significance of incidental desmoids identified during surgery for familial adenomatous polyposis. Dis Colon Rectum 47: 334-338, 2004. 
5. Hallet M: NINDS myotatic reflex scale. Neurology 43: 2723, 1993.

6. Tejpar S, Nollet F, Li C, et al: Predominance of beta-catenin mutations and beta-catenin dysregulation in sporadic aggressive fibromatosis (desmoid tumor). Oncogene 18: 6615-6620, 1999.

7. Alman BA, Li C, Pajerski ME, Diaz-Cano S and Wolfe HJ: Increased beta-catenin protein and somatic APC mutations in sporadic aggressive fibromatoses (desmoid tumors). Am J Pathol 151: 329-334, 1997.

8. Aberle H, Bauer A, Stappert J, Kispert A and Kemler R: $\beta$-Catenin is a target for the ubiquitin-proteasome pathway. EMBO J 16: 3797-3804, 1997.

9. Rubinfeld B, Albert I, Porfiri E, Fiol C, Munemitsu S and Polakis P: Binding of GSK $3 \beta$ to the APC- $\beta$-catenin complex and regulation of complex assembly. Science 272: 1023-1026, 1996.

10. Ghanbari-Azarnier R, Sato S, Wei Q, Al-Jazrawe M and Alman BA: Targeting stem cell behavior in desmoid tumors (aggressive fibromatosis) by inhibiting hedgehog signaling. Neoplasia 15: 712-719, 2013.

11. Wu C, Nik-Amini S, Nadesan P, Stanford WL and Alman BA: Aggressive fibromatosis (desmoid tumor) is derived from mesenchymal progenitor cells. Cancer Res 70: 7690-7698, 2010.

12. Escobar C, Munker R, Thomas JO, Li BD and Burton GV: Update on desmoid tumors. Ann Oncol 23: 562-569, 2012.

13. Rhim JH, Kim JH, Moon KC, et al: Desmoid type fibromatosis in the head and neck: CT and MR imaging characteristics. Neuroradiology 55: 351-359, 2013.

14. Nishio J, Aoki M, Nabeshima K, Iwasaki H and Naito M: Imaging features of desmoid-type fibromatosis in the teres major muscle. In Vivo 27: 555-559, 2013.

15. Owens CL, Sharma R and Ali SZ: Deep fibromatosis (desmoid tumor): Cytopathologic characteristics, clinicoradiologic features and immunohistochemical findings on fine-needle aspiration. Cancer 111: 166-172, 2007.

16. Qi H, Cal Cin P, Hernandez JM, et al: Trisomies 8 and 20 in desmoid tumors. Cancer Genet Cytogenet 92: 147-149, 1996.

17. Fiore M, Rimareix F, Mariani L, et al: Desmoid-type fibromatosis: A front-line conservative approach to select patients for surgical treatment. Ann Surg Oncol 16: 1587-1593, 2009.

18. Bonvalot S, Eldweny H, Haddad V, et al: Extra-abdominal primary fibromatosis: Aggressive management could be avoided in a subgroup of patients. Eur J Surg Oncol 34: 462-468, 2008.
19. van Broekhoven DL, Verhoef C, Elias SG, et al: Local recurrence after surgery for primary extra-abdominal desmoid type fibromatosis. Br J Surg 100: 1214-1219, 2013.

20. Ballo MT, Zagars GK, Pollack A, Pisters PW and Pollack RA: Desmoid Tumor: Prognosis factors and outcome after surgery, radiation therapy, or combined surgery and radiation therapy. J Clin Oncol 17: 158-167, 1999.

21. Gluck I, Griffith KA, Biermann JS, Feng FY, Lucas DR and Ben-Josef E: Role of radiotherapy in the management of desmoid tumors. Int J Radiat Oncol Biol Phys 80: 787-792, 2011.

22. Shin SH, Ko KR, Cho SK, Choi YL and Seo SW: Surgical outcome of desmoid tumors: adjuvant radiotherapy delayed the recurrence, but did not affect long-term outcomes. J Surg Oncol 108: 28-33, 2013.

23. Klein WA, Miller HH, Anderson M and DeCosse JJ: The use of indomethacin, sulindac and tamoxifen for the treatment of desmoid tumors associated with familial polyposis. Cancer 60: 2863-2868, 1987.

24. Janinis J, Patriki M, Vini L, Aravantinos G and Whelan JS: The pharmacological treatment of aggressive fibromatosis: A systematic review. Ann Oncol 14: 181-190, 2003.

25. Devata S and Chugh R: Desmoid tumors: A comprehensive review of the evolving biology, unpredictable behavior and myriad of management options. Hematol Oncol Clin North Am 27: 989-1005, 2013

26. Sundaram M, Duffrin $H$, McGuire $M H$ and Vas W: Synchronous multicentric desmoid tumors (aggressive fibromatosis) of the extremities. Skeletal Radiol 17: 16-19, 1988.

27. Antal I, Szendroi M, Kovacs G, Nagykalnai T and Entz L: Multicentric extraabdominal desmoid tumor: a case report. J Cancer Res Clin Oncol 120: 190-193, 1994.

28. Watanabe K, Ogura G, Tajino T and Suzuki T: Extra-abdominal desmoid fibromatosis: Two familial cases with synchronous and metachronous multicentric hyalinizing nodules. Histopathology 41: 118-121, 2002.

29. Eccles DM, van der Lujit R, Breukel C, et al: Hereditary desmoid disease due to a frameshift mutation at codon 1924 of the APC gene. Am J Hum Genet 59: 1193-201, 1996. 